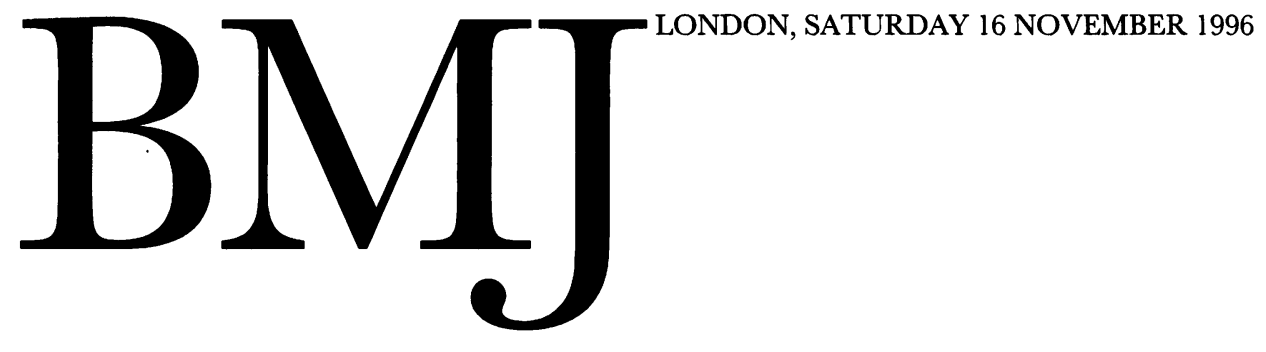

\title{
Depression and the menopause
}

\author{
Depression in a middle aged woman should not automatically be blamed on the menopause
}

The linking of the menopause with depression is pervasive in lay and medical discourse, despite limited supporting evidence. Historically, myths relating to menstrual taboos, as well as attitudes towards fertility and aging, have produced an image of the menopause as a time of physical and emotional decline. In the 19th century the menopause was thought to cause insanity, and it was only as recently as 1980 that the diagnosis of involutional melancholia was removed from the third edition of the Diagnostic and Statistical Manual of Mental Disorders. ${ }^{1}$ With the development of hormone replacement therapy, treatment of menopausal women shifted from the domains of psychiatry and psychoanalysis to gynaecology and endocrinology.

Initially prescribed for the treatment of vasomotor symptoms, hormone replacement therapy is now recommended for preventing osteoporosis and cardiovascular disease and improving quality of life. ${ }^{2}$ In the past few years interest has increased in the possible psychoactive properties of hormone replacement therapy. It has been proposed that oestrogen might cure "menopausal depression" and that it might offer an additional bonus of increased wellbeing or quality of life for non-depressed, healthy women.

Defining the menopause as an oestrogen deficiency disease implies that the cause of depression lies in a woman's hormones. This has important implications for the way middle aged and older women's views and behaviours are perceived in society. It is also relevant to the day to day clinical practice of many doctors, since menopausal women who seek medical help commonly present with a mixture of vasomotor and psychological symptoms. ${ }^{3}$

So, what is the evidence? Does the menopause cause deprèssion? In this week's BMF, Louise Nicol Smith (p 1229) presents a systematic review of epidemiological studies relating to this question, focusing on the issue of causality. ${ }^{4}$ As well as highlighting the considerable methodological problems in this area of research, the review concludes that there is no substantial evidence to support the view that a natural menopause causes depression. Crosscultural, epidemiological, correlational (hormone levels and depression), and intervention studies are all relevant to the relationship between menopause and depression, and are briefly outlined below.

Crosscultural research provides examples of communities in which women have positive attitudes to the menopause and report few or no symptoms. ${ }^{5}$ These suggest that the psychological impact of the menopause is influenced by the social values ascribed to older women and the roles available to them, as well as cultural differences affecting lifestyle factors, such as diet and exercise. ${ }^{6}$
As for epidemiological studies, several were carried out in the 1980s on middle aged women in North America and Europe. ${ }^{7-11}$ Two further studies are in progress in Australia and Sweden. In an attempt to overcome methodological problems inherent in earlier cross sectional designs, these studies used prospective samples and standardised measures. Overall, few changes were found to be attributable to the menopause other than hot flushes and night sweats. Though there was considerable variation between women in terms of wellbeing and depression, the prevalence of depression did not increase during the menopause in four of the five studies. Moreover, women of childbearing age tend to be more prone to depression. ${ }^{12}$

In the prospective studies, psychosocial factors were found to be the main predictors of depression during the menopause. ${ }^{9112}$ These included past depression, socioeconomic status, stressful life events such as bereavements, and negative beliefs about the menopause. The menopause seemed to have a more negative effect on women who previously believed that the menopause brings a host of physical and emotional problems. ${ }^{70}$ Marital and employment status as well as social support can moderate the effects of stress, as at other stages of life. Those who suffered chronic arthritis or thyroid problems were more prone to continued depression. ${ }^{8}$ Experiencing a longer menopause (at least 27 months) was associated with an increased but transitory risk of depression; this association seemed to be explained by increased exposure to vasomotor symptoms. ${ }^{7}$

Meanwhile, correlational studies have reached a fairly clear consensus that there is no link between oestrogen concentrations and depression. ${ }^{13}$ Further research is needed to explore the concurrent relations between hormone levels, vasomotor symptoms, and depressed mood in prospective studies with frequent repeated measures across the menopause transition.

Finally, intervention trials on the effects of hormone replacement therapy on depression and wellbeing are beset by methodological problems. They vary in the samples studied and the type and dosage of oestrogen regimens. Placebo effects are large, and few studies have controlled for the secondary effects of reduction in vasomotor symptoms. Though some studies have found improvements in mood, others have not. Overall, there is no conclusive evidence that hormone replacement therapy improves depression in women seeking help for menopausal problems over and above placebo effects. There is some evidence of a small improvement in wellbeing in selected, healthy women, particularly when higher doses are used. $^{13}{ }^{14}$ Again, carefully designed studies are needed to disentangle the effects of placebo, relief from vasomotor symp- 
toms, and the possible psychoactive properties of oestrogen However, even if hormone replacement therapy increases wellbeing or improves mood, this would not necessarily mean that depression is caused by low levels of oestrogen.

Taken together the above findings suggest that to attribute depression in a middle aged woman automatically to the menopause is overly simplistic and usually unjustified. The menopause has a psychological, social, and cultural, as well as a biological importance. On the biological level there is more evidence in support of prolonged or severe vasomotor symptoms causing distress than hormone levels. Nor should we forget that for most women the menopause is not a major crisis and that many feel relieved to be free from menstrual periods and the possibility of pregnancy.

When a middle aged woman seeks help for depression several possible causes need to be considered. For example, any stresses or conflicts in her life, bereavements, lack of social

1 American Psychiatric Association. Committee on Nomenclature and Statistics. Diagnostic and statistical manual of mental disorders. Washington DC: American Psychiatric Association, 1987.

2 Birkhauser $\mathrm{MH}$. Contraindications today, indications tomorrow. In: Birkhauser $\mathrm{MH}$ Rozenbaum H, ed. Menopause: European consensus development conference. Paris: ESKA Rozenbaum H,
Editions, 1996.

3 Morse CA, Smith A, Dennerstein L, Green A, Hopper J, Berger H. The treatment seeking woman at menopause. Maturitas 1994;18:161-73.

4 Nicol Smith L. Causality, menopause and depression: a critical review of the literature. $B M Y$ 1996;313:1129-32.

5 Flint M. The menopause: reward or punishment? Psychosomatics 1975;16:161-3.

6 Beyenne Y. Cultural significance and physical manifestations of menopause, a biocultural analysis. Cult Med Soc 1986;10:47-71.

7 Avis NE, Brambilla D, McKinlay SM, Vass K. A longitudinal analysis of the association between menopause and depression. Ann Epidemiol 1994;4:214-20. support, ill health, as well as severe or prolonged vasomotor symptoms that can lead to sleeplessness. She may feel uncertain about the effect of the menopause, have concerns about the physical and social consequences of aging, or her self esteem may be affected by a culture that values youth and reproductive capacity. In my experience a group setting can provide a useful place for women to discuss these issues, to reappraise past events and achievements, and to look to the future. Finally, to attribute depression to the menopause implies a hormonal cause, ignores psychosocial factors, and may indirectly promote the negative beliefs that have been found in epidemiological studies to predict depressed mood in middle aged women.

MYRA S HUNTER Clinical psychologist

University College London,

London WC1E 6BT

8 Kaufert PA, Gilbert P, Tate R. The Manitoba project; a re-examination of the link between menopause and depression. Maturitas 1992;14:143-56.

Holte A. Influences of natural menopause on health complaints; a prospective study of healthy Norwegian women. Maturitas 1992;14:127-41.

10 Hunter MS. The SE England longitudinal study of the climacteric and postmenopause. Maturitas 1992;14:117-26.

-11 Matthews KA, Wing RR, Kuller LH. Influences of natural menopause on psychological characteristics and symptoms of middle-aged healthy women. I Consult Clin Psychol 1990;58: 345-63.

12 Andrews B, Brown G. Stability and change in low self esteem: the role of psychosocial factors. Psychol Med 1995;25:1-9.

13 Hunter MS. Mental changes: are they due to the menopause? In: Birkhauser MH, Rozenbaum H, eds. Menopause: European consensus development conference. Paris: ESKA Editions, 1996. 14 Pearce J, Hawton K, Blake F. Psychological and sexual symptoms associated with the menopause and the effects of hormone replacement therapy: a review. $\mathrm{Br} \mathcal{F}$ Psychia 1995;167:163-73.

\section{Pet birds and lung cancer}

\section{Now no evidence of a link}

The question of whether keeping pet birds increases the risk of lung cancer was first raised by Holst. ${ }^{12}$ Having noted a higher occurrence of lung cancer among bird owners in a Dutch general practice population, he and colleagues carried out a case control study comparing hospital patients with community matched controls and showed a 6.7 -fold increase in risk. ${ }^{3}$ Two subsequent studies produced further evidence in support of this observation: one reported a twofold increase in risk of lung cancer in relation to exposure to pet birds in a German population, ${ }^{4}$ while the other, from Scotland, showed no significant association with exposure to pet birds in general but a 3.5-fold increase in risk of lung cancer in those who kept pigeons. ${ }^{5}$

At the time, we argued that, although of great importance if valid, these observations might have arisen from residual confounding by cigarette smoking. ${ }^{6}$ All of the case-control studies had controlled for smoking in their analysis but categorised cases and controls as smokers or non-smokers; the degree of smoking was not analysed quantitatively. In the ensuing correspondence, the authors of the two more recent reports argued that residual confounding was unlikely to have been a major influence because smoking histories seemed to have been similar in cases and controls. ${ }^{78}$ Others pointed out further methodological and confounding factors that might also have contributed to false positive results. ${ }^{910}$ In the end it was probably fair to conclude that the relation between bird keeping and lung cancer needed to be assessed further, in studies with sufficient power and appropriate measures of confounding variables to separate out the independent effects involved.

Two such studies, one from Missouri and the other from Sweden, are published in this issue of the $B M \mathcal{F}$ (pp 1233, 1236)..$^{112}$ Like their predecessors, both are case-control studies, but their strengths are that, with 652 and 380 cases respectively, they are considerably more powerful; they have ascertained incident cases only and thus avoided potential survival bias in relation to bird keeping in prevalent cases; they have measured smoking quantitatively and used this quantitative information in their analysis; and they have controlled for confounding by diet and socioeconomic status. They found no evidence whatever of an increase in risk of lung cancer in relation to any measure of bird keeping or exposure. Indeed, in some of the subgroups of exposure to pet or farm birds the risk of lung cancer was significantly reduced. The Missouri study is limited to women only, ${ }^{11}$ but the Swedish study includes both sexes and shows no evidence of a gender difference in effect. ${ }^{12}$ While there are inevitable potential criticisms of both studies, which the authors acknowledge, in our view none of these is likely to have resulted in odds ratios so close to unity in both reports. We therefore accept the authors' conclusion that keeping domestic birds does not seem to be associated with any excess risk of lung cancer.

So what is the explanation for the discrepancy between these new findings and the previous evidence? We argue, as before, that residual confounding by smoking is highly likely to have distorted the risk estimates in previous studies. In the new Swedish study the estimated odds ratios for lung cancer in current smokers of 20 cigarettes a day were about 32 and 43 respectively for men and women, ratios which far exceed the estimated effect of keeping pet birds in all earlier investigations. In the presence of effects so strong, the potential for bias in estimating the influence of other exposures even loosely related to smoking is substantial. An alternative explanation advanced by the authors is that exposure to pet birds in the populations they have studied is somehow different in intensity or in terms of associated exposures such 